\title{
Correspondence
}

\section{Prominent myalgia-an important clue in the diagnosis of a muscle disorder}

A developmentally normal, 15-year-old boy presented with complaint of gait abnormalities since 8 years of age. The child complained of difficulty in climbing stairs and running. He could not climb stairs without the support of railing. There was no problem with rising from sitting or lying down position. There was no history of upper limb weakness, difficulty in swallowing or breathing, restricted eye movements or diurnal variation of weakness. These complaints were very slowly progressive. He also complained of frequent myalgias (sometimes disabling), sometimes following exercise, affecting bilateral calf muscles and lower back. There was no stiffness or history of passing dark colored urine; and no significant family history was elicited.

Examination revealed normal spine examination and absence of muscle hypertrophy or atrophy. There was weakness at the hip flexors and abductors $(4 / 5)$ and neck flexors $(3 / 5)$. There was no facial, truncal or respiratory muscle weakness. The muscle stretch reflexes were normal. There was no muscle stiffness, myotonia, contractures or ligamentous laxity.

Serum creatine kinase (CK) was elevated (1248 U/L). His echocardiography was normal. The parents refused for muscle biopsy. Targeted sequencing analysis of congenital myopathy/dystrophy genes revealed a novel heterozygous missense mutation (c.13739T >A; p.Phe4580Tyr) in exon 94 of ryanodine receptor 1 gene $(R Y R I)$. Rehabilitation was initiated, risk of malignant hyperthermia was explained and genetic counselling was done.

Most cases with clinic-pathological features of central core disease are due to dominant missense mutations in the RYRl gene. The age of onset and phenotype is variable and include myopathies with permanent weakness and "induced" myopathies with anesthesia and non-anaesthesia related episodes of rhabdomyolysis or hyperCKemia. ${ }^{[1,2]}$ The reported case had very slowly progressive hip girdle weakness, prominent myalgias and mildly elevated serum CK. He did not have facial weakness or any orthopedic manifestation at present.

Myalgias with or without rhabdomyolysis are often associated or may be the only presenting feature of core myopathy. Dlamini et al ${ }^{[3]}$ identified RYR 1 mutations/variants in 14 out of 39 unrelated families with unexplained rhabdomyolysis and/or exertional myalgia. Myagias may also be a prominent feature in other muscle disorders (Table) ${ }^{[4]}$ Presentation at an interval after sustained exercise distinguishes RYR 1 related myalgia and rhabdomyolysis, for example from the glycogenoses, where early onset of symptoms and a second wind phenomenon are typical.

Table. Muscle disease associated with prominent myalgias

Congenital myopathies
Core myopathy
Congenital fibre type disproportion myopathy
Muscle dystrophies
Becker muscle dystrophy
Limb girdle muscle dystrophy 1C (caveolinopathy)
Limb girdle muscle dystrophy 2B (dysferlinopathy)
Limb girdle muscle dystrophy 2I (fukutin related protein deficiency)
Limb girdle muscle dystrophy 2L (anoctamine 5 deficiency)
Metabolic myopathies
Glycogenoses
Myoadenylatedeaminase deficiency
Lipidoses
Muscle phosphatidic acid phosphatase deficiency
Mitochondrial disorders

\section{Puneet Jain, Vineet Sehgal}

Department of Neonatal, Pediatric and Adolescent Medicine, Division of Pediatric Neurology, BL Kapur (BLK) Super Speciality Hospital, Pusa Road, New Delhi 110005, India; Division of Neurology, Department of Pediatrics, The Hospital for Sick Children, Toronto, Ontario, Canada (Jain P)

Department of Pediatrics, Max Super Speciality Hospital, Shalimar Bagh, New Delhi, India (Sehgal V)

Email:puneet_mpa@yahoo.com

\section{References}

1 Jungbluth H, Sewry CA, Muntoni F. Core myopathies. Semin Pediatr Neurol 2011;18:239-249.

2 Snoeck M, van Engelen BG, Küsters B, Lammens M, Meijer R, Molenaar JP, et al. RYR1-related myopathies: a wide spectrum of phenotypes throughout life. Eur J Neurol 2015;22:1094-1112.

3 Dlamini N, Voermans NC, Lillis S, Stewart K, Kamsteeg EJ, Drost $\mathrm{G}$, et al. Mutations in RYRI are a common cause of exertional myalgia and rhabdomyolysis. Neuromuscul Disord 2013;23:540-548.

4 Chan EK, Kornberg AJ, Ryan MM. A diagnostic approach to recurrent myalgia and rhabdomyolysis in children. Arch Dis Child 2015;100:793-797.

doi: 10.1007/s12519-017-0029-2 